\title{
3D Monte Carlo radiation transfer modelling of photodynamic therapy
}

\author{
C. Louise Campbell ${ }^{a}$, Craig Christison ${ }^{a}$, C. Tom A. Brown ${ }^{a}$, Kenneth Wood ${ }^{a}$, Ronan M. \\ Valentine $^{b}$ and Harry Moseley ${ }^{b}$ \\ ${ }^{a}$ SUPA, School of Physics and Astronomy, University of St Andrews, UK; \\ ${ }^{b}$ Photobiology Unit, Ninewells Hospital and Medical School, University of Dundee, UK
}

\begin{abstract}
The effects of ageing and skin type on Photodynamic Therapy (PDT) for different treatment methods have been theoretically investigated. A multilayered Monte Carlo Radiation Transfer model is presented where both daylight activated PDT and conventional PDT are compared. It was found that light penetrates deeper through older skin with a lighter complexion, which translates into a deeper effective treatment depth. The effect of ageing was found to be larger for darker skin types. The investigation further strengthens the usage of daylight as a potential light source for PDT where effective treatment depths of about $2 \mathrm{~mm}$ can be achieved.
\end{abstract}

Keywords: Monte Carlo Radiation Transfer modelling of Photodynamic Therapy, Theoretical investigation of Photodynamic therapy, Daylight Activated Photodynamic Therapy, Tissue Optics, Skin Ageing

\section{INTRODUCTION}

Photodynamic therapy (PDT) is an efficient treatment method for non-melanoma skin cancer (NMSC) and precancerous skin lesions. The treatment method has proved very effective with a good cosmetic outcome and is widely used for treating, for example, Basal Cell Carcinoma (BCC) and Aktinic Keratosis (AK). PDT combines light, molecular oxygen and a photosensitive molecule to kill targeted tumour cells. Topical PDT uses a prodrug in the form of a cream (containing either 5-aminolaevulinic acid (ALA) or methyl aminolevulinate (MAL)), which is converted within the cancerous cells to the photosensitive molecule Protoporphyrin IX (PpIX). The high selective accumulation of PpIX in the tumour tissue leads to a selective treatment where the surrounding healthy tissue is left relatively unaffected by the illuminating light. ${ }^{1-3}$

Light is absorbed by PpIX over a wide range of wavelengths (figure 1). Therefore it is possible to use different types of treatment light sources. Here we investigate two different sources: an artificial light source (Aktilite, Photocure ASA, Oslo, Norway ${ }^{4}$ ) and a natural light source (daylight, figure 1). Daylight has recently been investigated as a potential light source for PDT. ${ }^{5}$ This treatment method allows for larger areas to be treated and studies show that daylight activated PDT is associated with lower experienced pain and is the preferred choice of treatment. ${ }^{6}$ Clinical studies around the world have shown that daylight PDT results in high efficacy when applied to superficial skin lesions. ${ }^{7}$

During PDT the same treatment parameters are applied, independent of age and skin type. The aim of this work is to investigate how the ageing of skin and different skin types affects the response during PDT. Mathematical computer models are used to theoretically investigate the dynamics of the treatment and the light interaction in the skin tissue. ${ }^{8,9}$ A multilayered three dimensional (3D) Monte Carlo Radiation Transfer (MCRT) model is presented, where the properties within each separate layer vary to represent different ages and skin types. Daylight PDT and conventional PDT are compared for these simulated treatment conditions.

Further author information: (Send correspondence to C L Campbell)

C L Campbell: E-mail: clc57@st-andrews.ac.uk, Telephone: 15051231234

Biophotonics South America, edited by Cristina Kurachi, Katarina Svanberg, Bruce J. Tromberg,

Vanderlei Salvador Bagnato, Proc. of SPIE Vol. 9531, 95311H · ( 2015 SPIE

CCC code: $1605-7422 / 15 / \$ 18 \cdot$ doi: $10.1117 / 12.2180744$

Proc. of SPIE Vol. $953195311 \mathrm{H}-1$ 

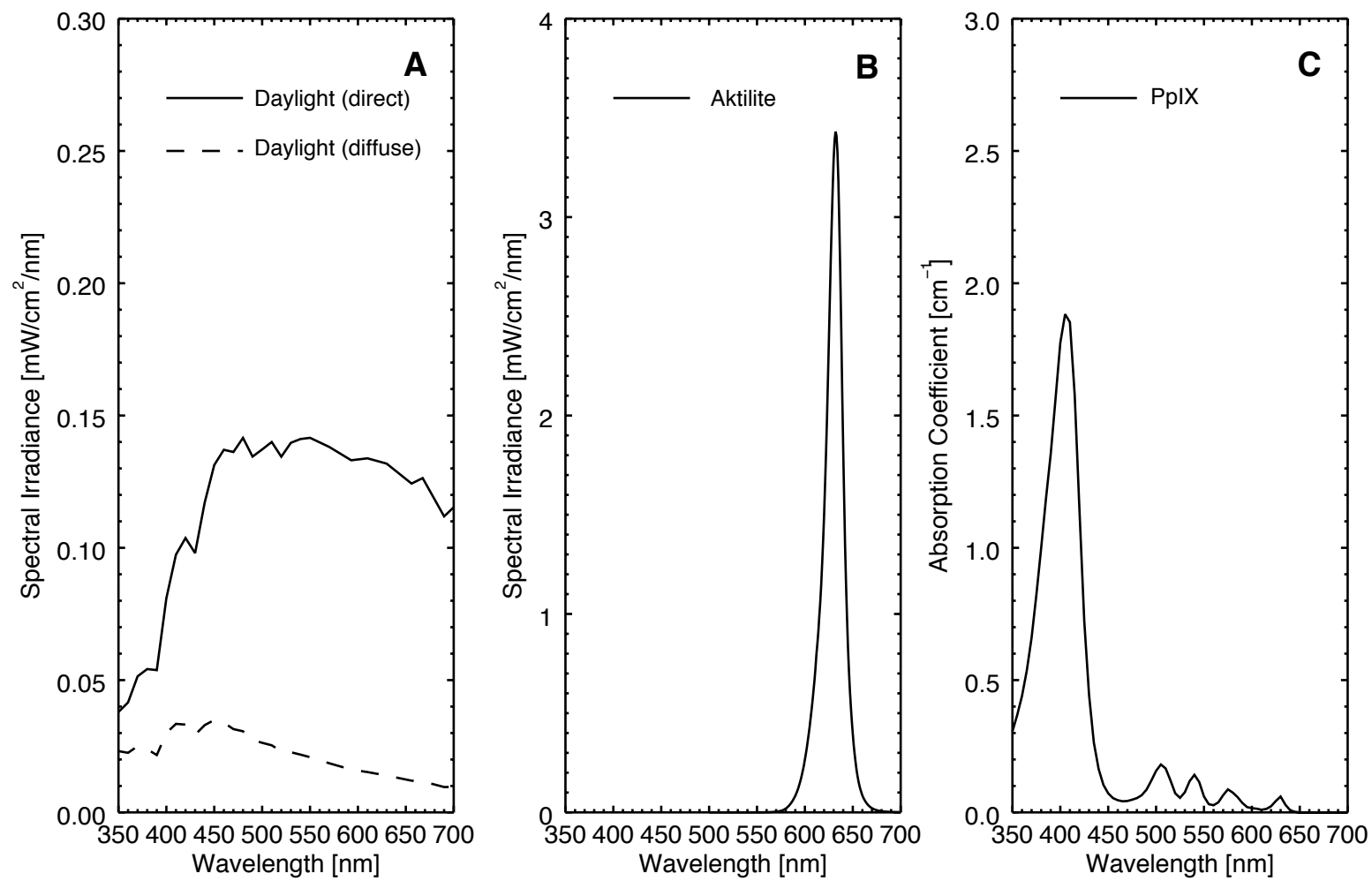

Figure 1: A) Spectral irradiance for daylight, where both the direct and diffuse component of the spectrum are displayed. B) The spectrum for the Aktilite, which is a narrow band light source centred at $630 \mathrm{~nm}$. C) The absorption spectrum of PpIX for an assumed uniform initial number density of PpIX molecules of $1.62 \times 10^{14} \mathrm{~cm}^{-3} \cdot 12,13$

\subsection{Anatomy of skin and skin ageing}

The human skin can be divided into several different layers with differing structure and optical properties. The structure and components of the skin, and therefore also the function of the skin, varies with age, race, gender and individual. The model presented here includes 5 separate layers of different tissue types (figure 2 ). The outermost layer is the epidermis which can be divided into two sub-layers; the stratum corneum (SC) and the living epidermis (LE). The second main layer is the dermis, which can also be divided into two layers, the papillary dermis $(\mathrm{PD})$ and the reticular dermis $(\mathrm{RD})$. The final layer that is included in the model is the hypodermis (HD). ${ }^{10,11}$

The composition and structure of skin changes with age and appropriate care of elderly skin has great medical importance. ${ }^{11}$ The ageing of skin is a complex procedure that is due to both endogenous and exogenous factors. The endogenous factors include the physiological and chronological changes that affect skin as it ages. The exogenous factors that contribute to ageing include ultraviolet (UV) radiation, toxins and infectious agents that can lead to skin damage from DNA changes. ${ }^{14}$ Due to the medical importance it is important to understand 


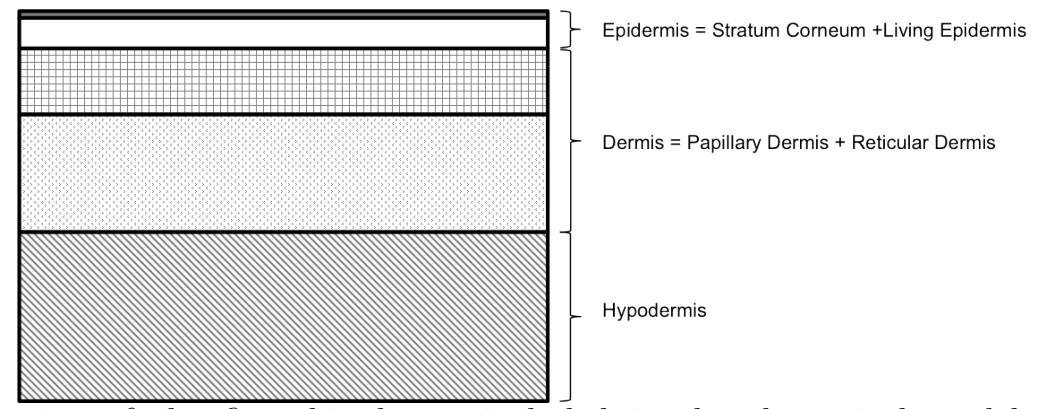

Figure 2: Representation of the five skin layers included in the theoretical model. The thickness and blood/melanin content within the layers were adjusted accordingly to ageing and different skin types.

how ageing changes the response to light based treatment methods such as PDT. The work presented here follows on from recently published work by Iglesias-Guitian et $\mathrm{al}^{15}$ where the main changes in composition and structure due to ageing are included. The changes that are considered are thinning of the different skin layers (approximately 6-16\% reduction per decade ${ }^{16}$ ) and reduction of the main absorbing components in the skin: melanin (epidermis, approximately $8 \%$ per decade ${ }^{17}$ ) and blood (dermis, approximately $6-10 \%$ per decade ${ }^{18}$ ). As well as ageing, different skin types, i.e. levels of pigmentation, ${ }^{19}$ are investigated where the melanin content is adjusted appropriately.

\section{METHODS}

The radiative transfer equation (RTE) describes the photo-propagation through scattering media, such as skin tissue. ${ }^{20}$ However, the equation is difficult to solve and therefore several approximations have been developed to determine the light distribution within the medium. MCRT is a numerical technique that utilises the probabilistic nature of photon interactions to determine the distribution of light in tissue and is therefore an appropriate tool for simulating PDT. ${ }^{9,21}$ The MCRT code presented here has been developed from a publicly available code, originally developed for applications in astronomy. ${ }^{12,22}$

\subsection{Monte Carlo radiation transfer}

The MCRT code tracks power packets (hereafter referred to as photons) through a 3D linear Cartesian grid containing voxels, all with individual optical properties. This allows for complex 3D structures to be included in the model. The photons are launched from the top of the grid and each photon is assigned an initial direction and wavelength depending on the illuminating light source. The wavelengths are sampled such that the probability distribution function of the irradiance, and therefore the illuminating spectra, are reproduced. The photons are tracked until they are absorbed or scattered out of the simulation grid. As they travel through the grid they contribute to counters that contain the information about the distribution and energy deposition of the photons within the skin tissue.

\subsection{Code validation}

The code was validated using an experimental approach where the aim was to successfully reproduce real measurements with the MCRT code. The fluorescence signal was obtained from a solution containing Coproporphyrin III, distilled water and a varied concentration of Intralipid 20\% (Fresenius Kabi, Germany). Intralipid is highly scattering and is frequently used for studying light propagation through turbid media. The fluorescence signal generated by the Coproporphyrin was recorded using an optical biopsy system (OBS), comprising a $405 \mathrm{~nm}$ laser diode for excitation and a spectrometer (USB 2000, Ocean Optics) for detection. The same $600 \mu \mathrm{m}$ diameterer fiber probe is used for delivery of the excitation radiation and collection of the fluorescence signal. ${ }^{13}$ The experimental set up was simulated using the MCRT code with appropriate optical properties and dimensions. A cube with the dimensions $10 \mathrm{~mm} \times 10 \mathrm{~mm} \times 10 \mathrm{~mm}$ was simulated to represent the $10 \mathrm{~mm} \times 10 \mathrm{~mm}$ cuvette used in the lab. The illuminating and collecting region was simulated to match the diameter and numerical aperture of the probe. It was assumed the anisotropy factor was constant for all wavelengths and was kept at $0.7 .{ }^{23}$ The albedo 

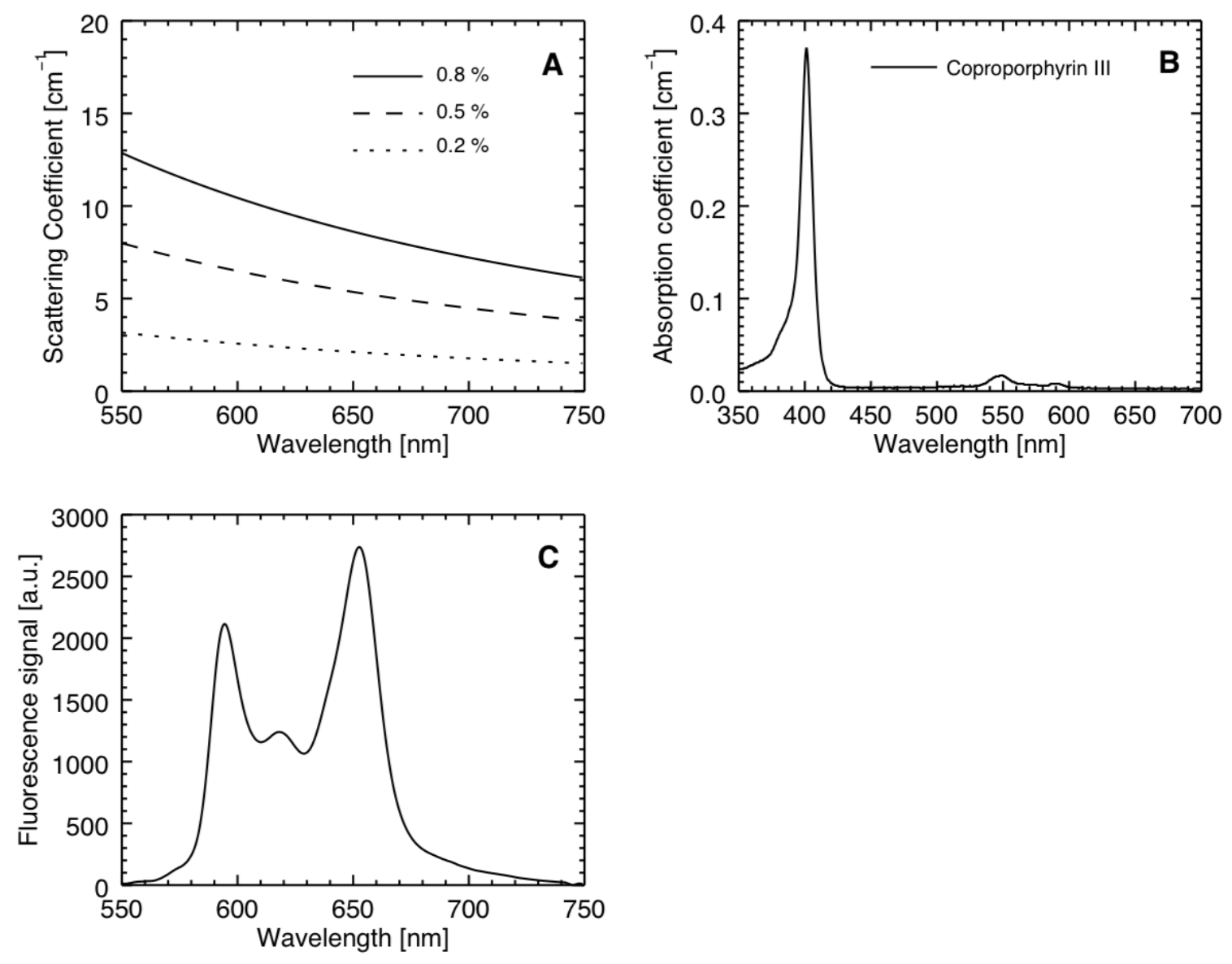

Figure 3: A) Scattering properties used in the simulations. The different concentrations corresponds to different concentrations of Intralipid 20 \% . B) Absorption spectrum of Coproporphyrin III. C) Fluorescence spectrum of Coproporphyrin III in the range $500 \mathrm{~nm}$ to $750 \mathrm{~nm}$.

for the Intralipid was assumed to be 0.999 due to its highly scattering features. ${ }^{24}$ The scattering properties of the Intralipid are assumed to be scaled with the Intralipid concentration and the scattering is assumed to scale equally over the wavelength range. The scattering properties for Intralipid $20 \%$ are known ${ }^{25}$ and the scaling factor used follow a power law function based on previous work ${ }^{24}$ (figure 3 a). The measured absorption of the Coproporphyrin is shown in figure $3 \mathrm{~b}$. The fluorescence spectrum, used in the MCRT code, was recorded using OBS measurements from a sample of pure Coproporphyrin (figure $3 \mathrm{c}$ ). The measured and simulated fluorescence of the Coproporphyrin III with varied concentration of Intralipid $20 \%$ produced using the MCRT code is shown in figure 4. The change in the strength of the fluorescence signal is due to variations in the scattering properties. An increased scattering causes more diffusion of the fluorescence signal. The collection geometry (diameter and numerical aperture) remains the same in all cases so increasing the scattering causes a reduction in the overall fluorescence signal as can be clearly seen in the experimental measurements (solid lines) in figure 4 . This feature due to the variation in Intralipid concentration was reproduced successfully by the developed MCRT code as shown by the dashed lines in figure 4. 

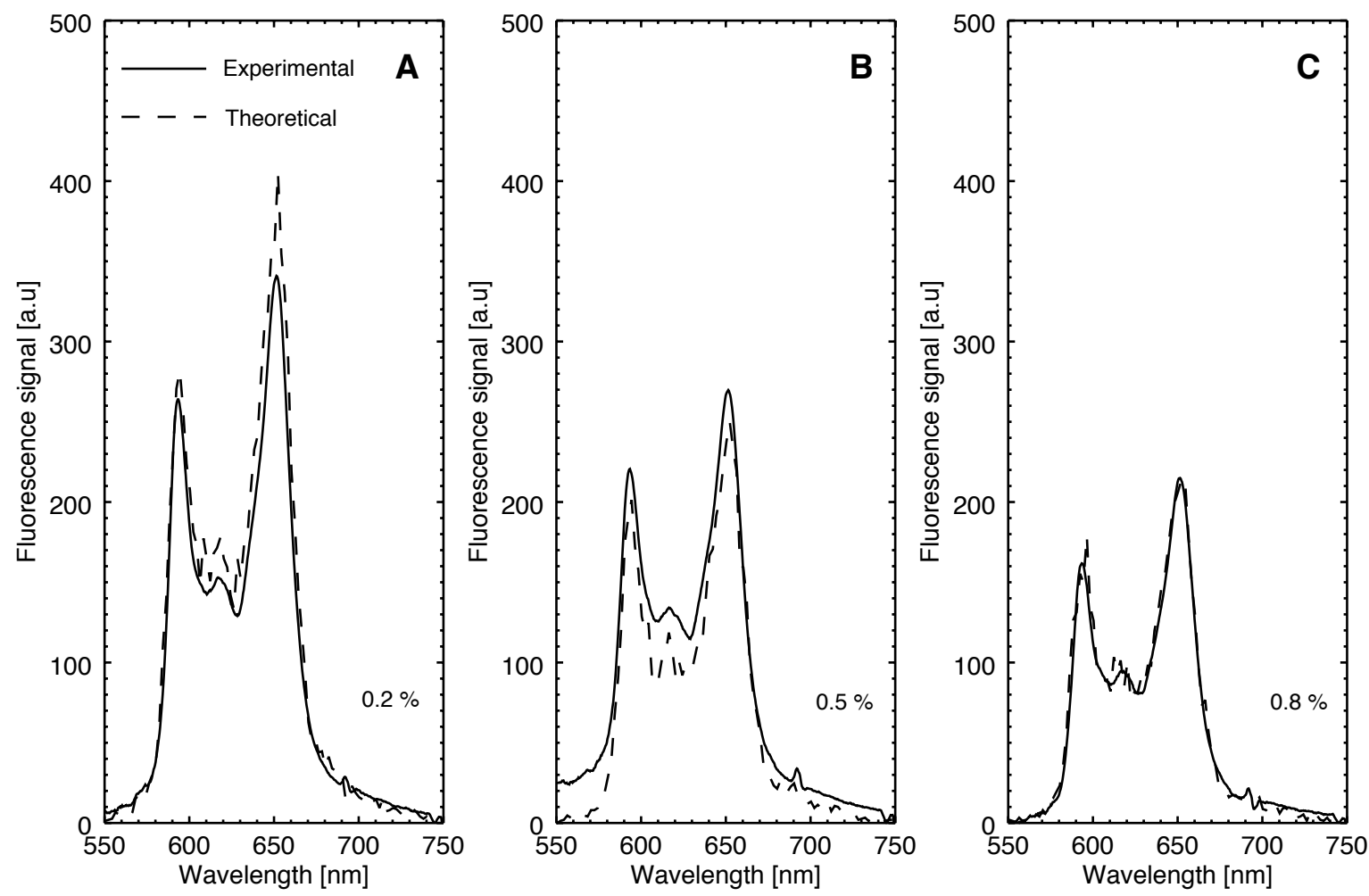

Figure 4: Figure comparing experimental and theoretical results of the fluorescence signal, in arbitrary units (a.u.), generated by Coproporphyrin III in a solution with varied concentration of Intralipid $20 \%$. A) $0.2 \%$ of Intralipid 20\%, B) $0.5 \%$ of Intralipid $20 \%$ and C) $0.8 \%$ of Intralipid $20 \%$. 
Table 1: Thicknesses for the investigated layers were included in the skin model to represent ageing. ${ }^{15}$

\begin{tabular}{|l|lll|}
\hline \multicolumn{3}{|c|}{ Thickness (mm) } \\
\hline Skin layer & 30 & 55 & 80 \\
\hline Stratum Corneum & 0.02 & 0.02 & 0.02 \\
Living Epidermis & 0.08 & 0.07 & 0.06 \\
Papillary Dermis & 0.18 & 0.15 & 0.13 \\
Reticular Dermis & 1.82 & 1.55 & 1.27 \\
Hypodermis & 5.9 & 5.9 & 5.9 \\
\hline
\end{tabular}

\subsection{Code set up}

The modelled skin tissue includes 5 separate layers where the thickness and composition of the different layers were changed appropriately to represent ageing (table 1) for different skin types (table 2). The dimensions of the simulation is set to be $5 \mathrm{~mm} \times 5 \mathrm{~mm} \times 5 \mathrm{~mm}$ and repeated boundary conditions were adopted to represent a semi-infinite tissue slab. This model allows for photobleaching to be taken into account which changes the distribution and concentration of PpIX during the light illumination. The PpIX photobleaching depends on both the fluence rate, as well as the initial concentration of PpIX. ${ }^{26,27}$ The equation that describes the photobleaching assumes an exponential decay of PpIX, which is wavelength dependent ${ }^{5}$ and an unlimited oxygen supply. To be able to quantify the treatment and to enable comparison of different treatment methods, the photodynamic dose (PDD) is determined within the code. The PDD is defined as the number of absorbed photons (by the photosensitiser) per $\mathrm{cm}^{3}$. Following methods by Farrell et al the PDD was considered to be proportional to the singlet oxygen production. ${ }^{27} \mathrm{~A}$ toxic threshold is included to determine the limit of effective treatment. A threshold of $8.6 \times 10^{17}$ photons $\mathrm{cm}^{-3}$ has previously been determined from measurements of Photofrin in liver tissue and has been adopted here for illustrative purposes to enable comparison between different treatment conditions and previously established models. ${ }^{26}$

Table 2: Input parameters were used for the melanin and heamoglobin levels for the Living Epidermis (LE), the Papillary Dermis (PD) and the Reticular Dermis (RD). These parameters were used to compute the optical properties of the different skin layers to represent different ages and skin types. The hypodermal layer is assumed to contain $5 \%$ of haemoglobin. The blood oxygen saturation was assumed to be $75 \%{ }^{15} 30,55$ and 80 corresponds to the age of the skin.

\begin{tabular}{|c|c|c|c|c|c|c|c|c|c|}
\hline \multicolumn{10}{|c|}{ Melanin and heamoglobin volume fraction (\%) } \\
\hline \multirow[t]{2}{*}{ Skin type } & \multicolumn{3}{|c|}{ Melanin (LE) } & \multicolumn{3}{|c|}{ Heamoglobin (PD) } & \multicolumn{3}{|c|}{ Heamoglobin (RD) } \\
\hline & 30 & 55 & 80 & 30 & 55 & 80 & 30 & 55 & 80 \\
\hline I & 1 & 0.8 & 0.6 & 6 & 3.6 & 1.2 & 4.5 & 2.73 & 0.9 \\
\hline II & 3 & 2.4 & 1.8 & 6 & 3.6 & 1.2 & 4.5 & 2.73 & 0.9 \\
\hline III & 5 & 4 & 3 & 6 & 3.6 & 1.2 & 4.5 & 2.73 & 0.9 \\
\hline IV & 10 & 8 & 6 & 6 & 3.6 & 1.2 & 4.5 & 2.73 & 0.9 \\
\hline V & 20 & 16 & 12 & 6 & 3.6 & 1.2 & 4.5 & 2.73 & 0.9 \\
\hline VI & 30 & 24 & 18 & 6 & 3.6 & 1.2 & 4.5 & 2.73 & 0.9 \\
\hline
\end{tabular}

\subsection{Optical properties}

To accurately model the light propagation through a medium, appropriate optical properties have to be assigned to the different grid cells. For the skin model the relevant parameters were varied so that skin ageing and skin types were appropriately represented (figure 5). To determine the absorption properties the main absorbing components were included as well as background absorption from human flesh. This allowed for adjustment of melanin and blood content to represent ageing and different skin types (table 2). The scattering properties were determined from power law functions fitted to experimental data where both Mie and Rayleigh scattering contributions are included. ${ }^{15,28-30}$

The Heyney-Greenstein phase function was adopted to describe the scattering directions within the skin tissue, ${ }^{31}$ where the anisotropy factor, $\mathrm{g}=0.62+\lambda \times 0.29 \times 10^{-3}$ was adopted $\left(\lambda\right.$ in nm). ${ }^{32}$ The refractive 

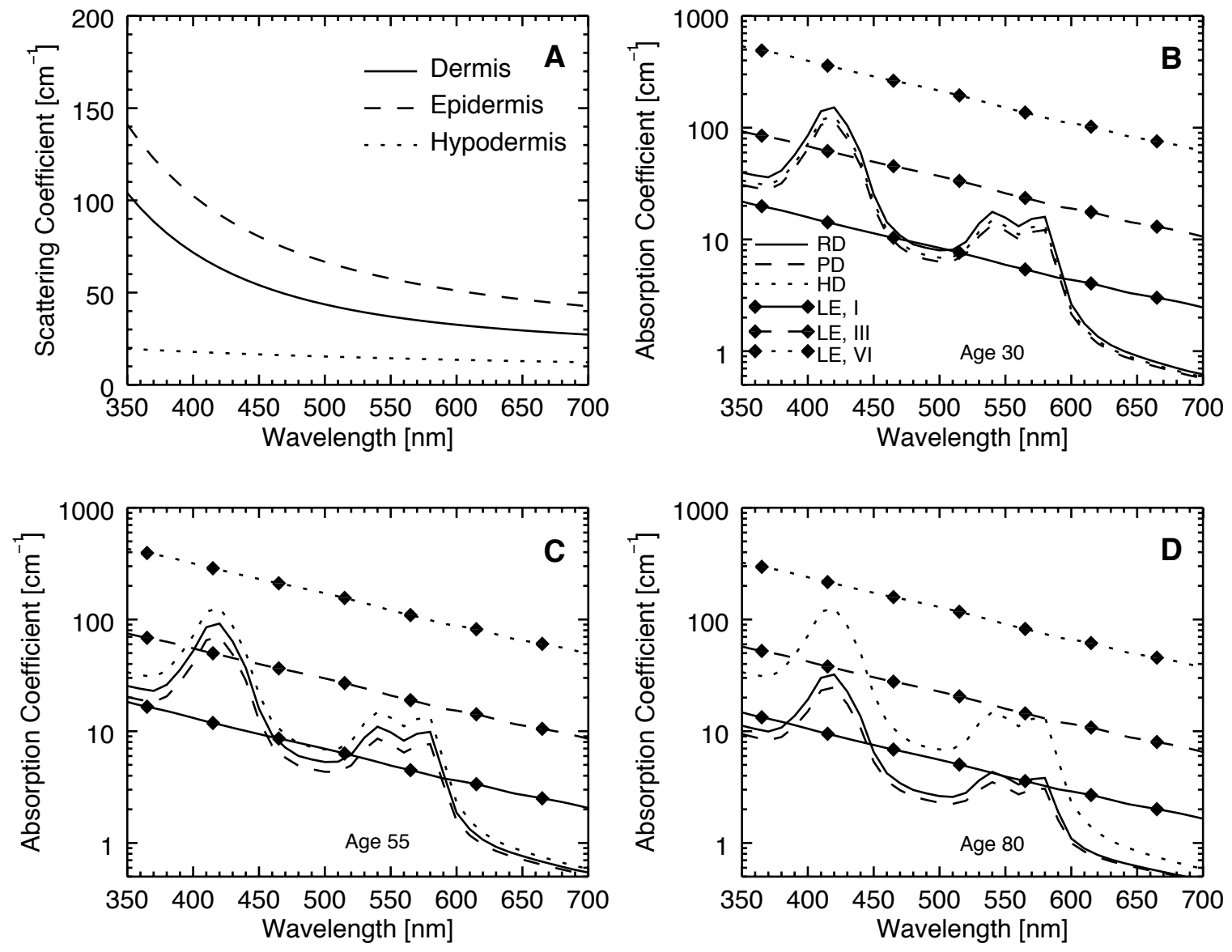

Figure 5: Figure showing the optical properties adopted in the MCRT model to represent different ages and skin types. A) Scattering coefficients for the three main layers. These are assumed to be the same both for different skin types and different ages. B) The absorption coefficients at age 30 where the reticular dermis (RD), papillary dermis (PD) and hypodermis (HD) are assumed to be constant for different skin types (not for different ages). The Epidermal layers (both Stratum Corneum and Living Epidermis (LE)) are assumed to have uniform absorption properties. However the melanin concentration will vary with skin type, and therefore the absorption will change accordingly. Three skin types are displayed to demonstrate the effect of change in pigmentation. C) The same absorption properties (same labelling), at an age of 55 years. D) Absorption coefficients at an age of 80 years. 

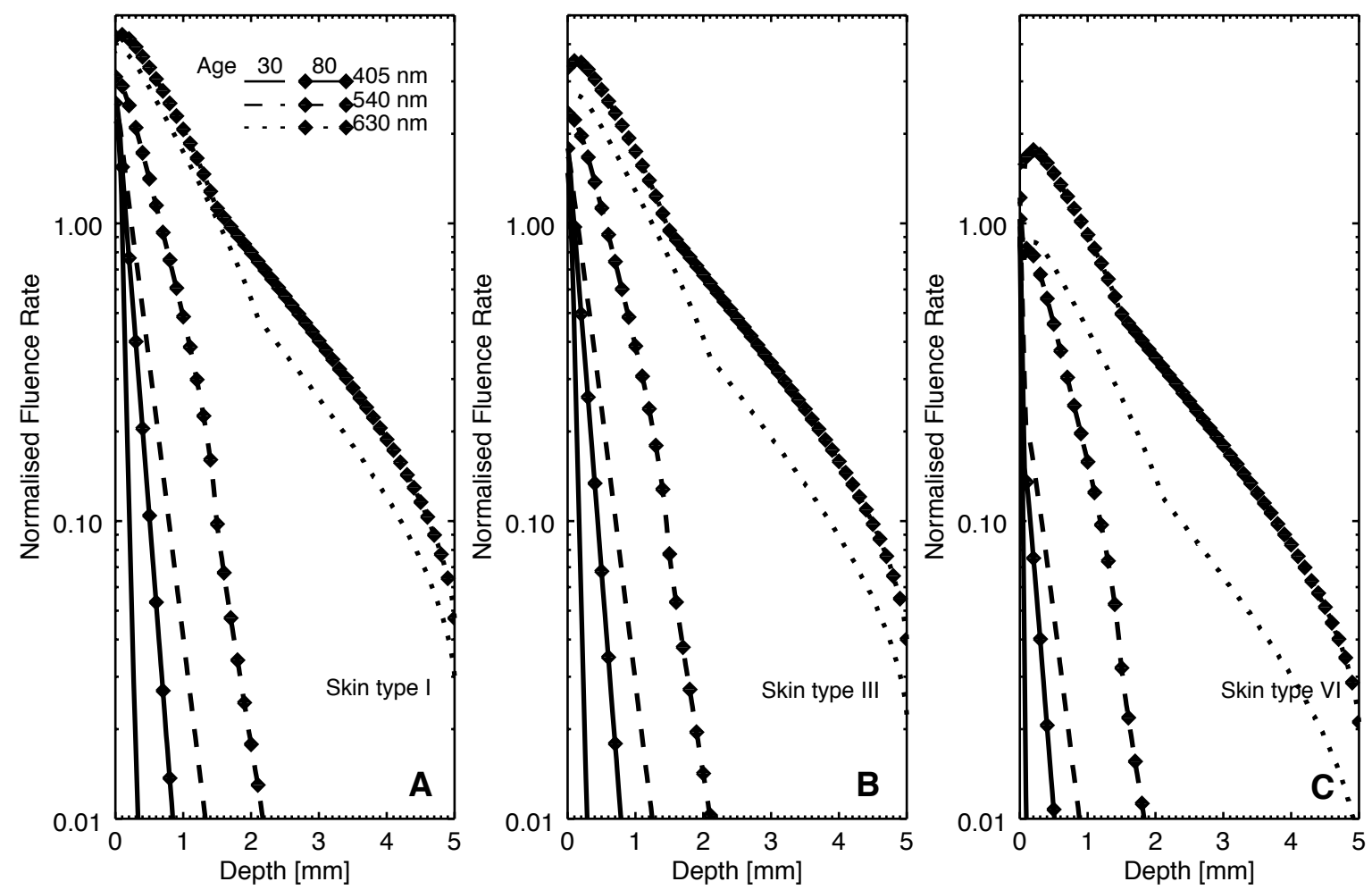

Figure 6: Figure showing fluence rate as a function of depth for different ages and skin types. Each figure demonstrates the effect of ageing, where the optical properties for an age of 30 (no diamonds) and 80 (diamonds) years are incorporated. Three wavelengths are investigates: $405 \mathrm{~nm}$ (solid line), $540 \mathrm{~nm}$ (dashed lines) and 630 nm (dotted line). A) Skin type I. B) Skin type III, C) Skin type VI .

index for the different layers were assumed to be uniform throughout the simulated region $(\mathrm{n}=1.38)$. The initial distribution of the PpIX was also assumed to be uniform. As the concentration of PpIX changes, due to photobleaching, the absorption properties of the PpIX change accordingly.

\subsection{Light sources}

The distribution of light under the surface of the skin depends on the spectrum and angular distribution of the incident light. The work presented here adopts accepted methods to produce both direct and diffuse components of the daylight. ${ }^{33,34}$ By decomposing the relative components it is possible to simulate daylight PDT for different weather conditions. For an overcast day it is assumed that all of the illuminating light comes from the diffuse component, whilst during a clear day only about $20 \%$ of the illuminating spectrum is assumed to be diffuse light. Here daylight activated PDT is investigated and compared with the conventional Aktilite light source. The irradiance for the Aktilite is $82 \mathrm{~mW} \mathrm{~cm}^{-2}$, for daylight PDT during a clear summer day the irradiance was determined to be $41 \mathrm{~mW} \mathrm{~cm}^{-2}$ and for an overcast summer day the irradiance was determined to be $8 \mathrm{~mW} \mathrm{~cm}^{-2}$. 

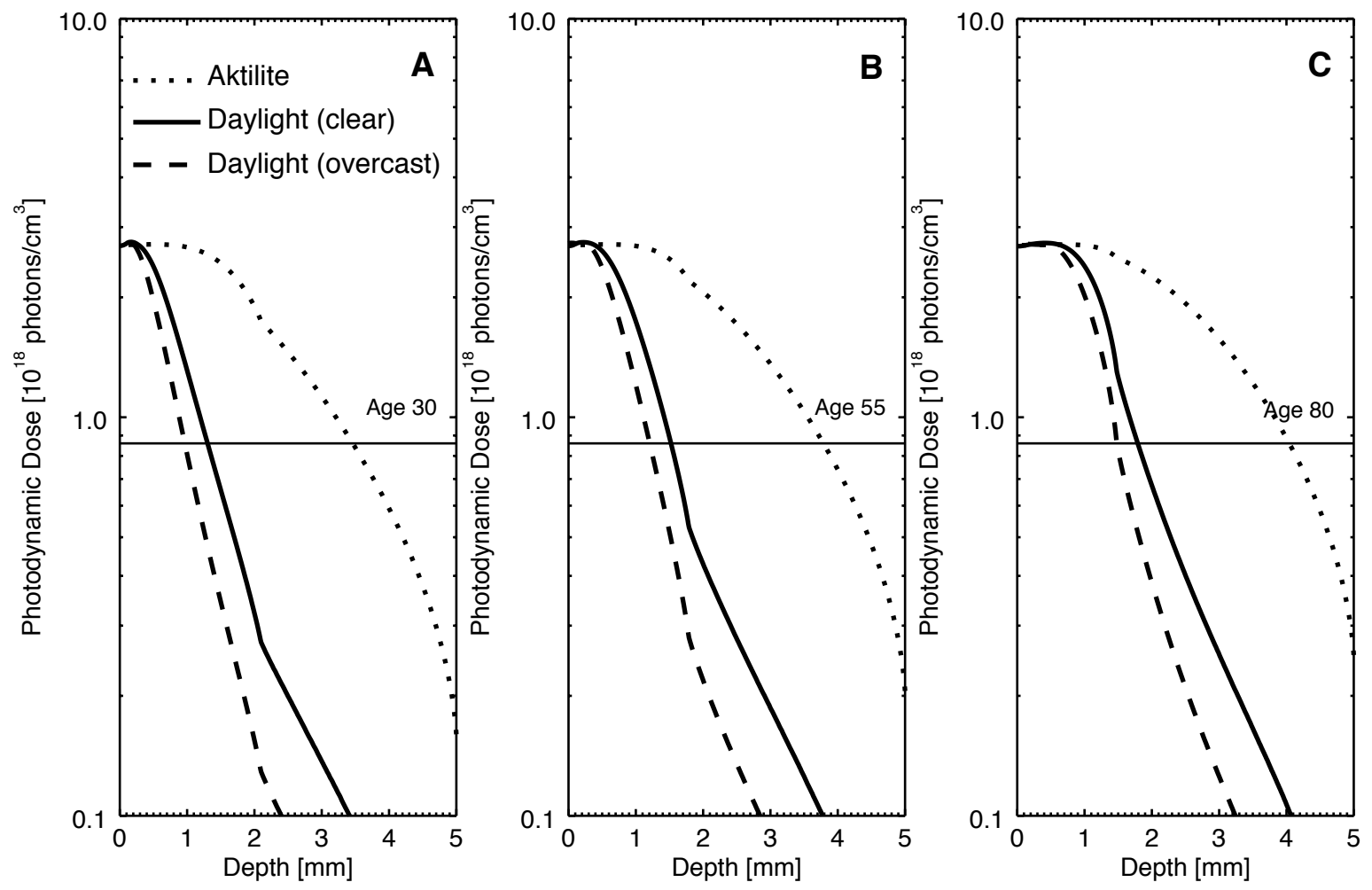

Figure 7: Photodynamic dose corresponding to $75 \mathrm{Jcm}^{-2}$ for the different light sources at age A) 30, B) 55 and C) 80, for skin type I. Three light sources are compered: daylight for clear conditions (solid line), daylight during overcast (dashed line) and the artificial light source, Aktilite (dotted line). The results show the change in effective penetration depth with age where the horizontal line corresponds to the adopted toxic threshold.

These values for the sunlight are equivalent to those observed at noon in summer at a latitude of 56 degrees North. ${ }^{5}$

\section{RESULTS}

The penetration of light depends strongly upon the illuminating wavelength due to the wavelength dependency of the optical properties (figure 5). To demonstrate this the fluence rate was generated for three different wavelengths (405, 540 and $630 \mathrm{~nm}$ ), for different skin types (I,III and VI) at different ages (30,55 and 80 years) (figure 6). The fluence rate describes the distribution of light within the skin tissue and indicates the effect of the investigated changes on the penetration depth. The fluence rate reduces with skin type number and increases with age. The ageing reduces both the melanin content and blood content while the difference between different skin type is only represented with a change in melanin content. Ageing of skin therefore has a larger effect on the fluence rate at depth, however the change in melanin content is larger between different skin types than between the ages presented here. 

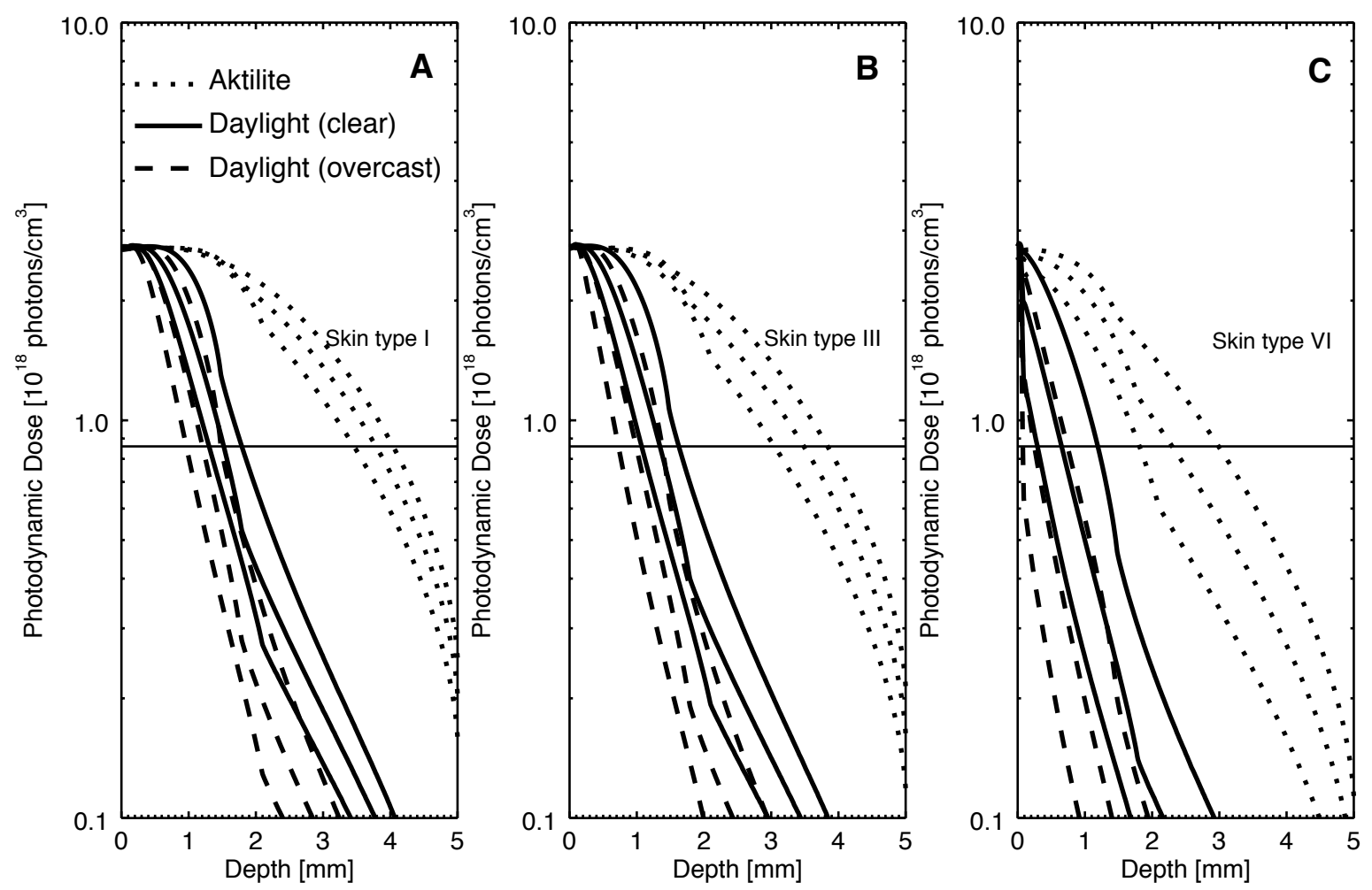

Figure 8: Figure comparing the effect due to ageing of the skin for different skin types: A) I, B) III and C) VI. Each figure contains the PDD at $75 \mathrm{Jcm}^{-2}$ for three different ages $(30,55$ and 80 years) for the three different light sources (daylight during clear conditions (solid line), daylight during overcast conditions (dashed) and the Aktilite (dotted)). The most shallow contribution corresponds to the youngest age, while the the deepest contribution corresponds to the oldest age (for all light sources investigated) 
The effects of skin type and ageing are summarised in figures 7 and 8. Figure 7 shows the PDD for a light dose corresponding to $75 \mathrm{~J} \mathrm{~cm}^{-2}$, which is a typical light dose delivered for conventional PDT. The effects for skin type I which is a common group affected by NMSC, are demonstrated. The effect of skin type, is investigated for the full light dose $\left(75 \mathrm{~J} \mathrm{~cm}^{-2}\right)$, for different ages (30,55 and 80 years) and skin types (I, III, VI) (figure 8). The effect of ageing, between 30 and 80 years, for skin type I corresponds to an effective treatment deapth of approximately $0.5 \mathrm{~mm}$. For skin type VI the same change corresponds to a approximately $1 \mathrm{~mm}$ of increase in treatment depth.

\section{DISCUSSION}

The results shown in figure 4 show that the MCRT code can be used to reproduce experimental measurements. The slight discrepancy between the theoretical and experimental results is due to uncertainty in the scattering properties of the Intralipid across a wide range of wavelengths but nevertheless shows a good agreement between out model and the experimental results.

Results shown in figures 6,7 and 8 demonstrate the effect of skin ageing on light interaction with different tissue types. The effective treatment depth is shown to increase with ageing and decrease with increasing skin type classification number. The results suggest that daylight is a potentially useful light source for treating superficial skin lesions for different ages and skin types, which agrees with previous clinical and theoretical investigations. The deeper treatment depth is shown to be associated with older patients of lighter complexion, which is also the most common patient group.

This investigation shows the dependence of treatment on different optical properties arising due to different ages and skin type. It is clear from these results that it is important to incorporate appropriate optical properties to enable accurate theoretical skin models to be developed. Since the range of published optical properties for skin tissue is large, ${ }^{28,35}$ this type of investigation is important to demonstrate the effect of light interaction with skin tissue as the optical properties are altered.

The model developed for this work only considers healthy tissue, where the same initial conditions for daylight and conventional PDT are assumed. Due to the limited availability of optical properties of tumour tissue, tumour structures have not been included. Future developments should include tumour tissue where the PpIX is confined.

Further developments of the MCRT code will lead to a more detailed investigation of the light interaction within skin tissue for different treatment conditions. The model presented here does not take into account the dryness, surface roughness or uneven pigmentation, which are other effects associated with ageing. ${ }^{11}$ However, these are factors that could be included in a future study. The techniques demonstrated here are also applicable in other areas where the range of ages and skin types is larger. One such area would be laser hair removal, where it would be of both interest and relevance to study a larger range of patient groups.

\section{ACKNOWLEDGMENTS}

We acknowledge the support of the UK Engineering and Physics Sciences Research Council (EPSRC) for funding through a studentship for C L Campbell as well as the Alfred Stewart Trust.

\section{REFERENCES}

[1] Wilson, B. and Patterson, M., "The physics, biophysics and technology of photodynamic therapy," Physics in Medicine and Biology 53(9), R61 (2008).

[2] Castano, A., Demidova, T., and Hamblin, M., "Mechanisms in photodynamic therapy: part one - photosensitizers, photochemistry and cellular localization," Photodiagnosis and Photodynamic Therapy 1(4), 279 -293 (2004).

[3] Darlenski, R. and Fluhr, J., "Photodynamic therapy in dermatology: past, present, and future," Journal of Biomedical Optics 18(6), 061208-061208 (2012).

[4] Moseley, H., "Light distribution and calibration of commercial pdt led arrays," Photochem. Photobiol. Sci. 4, 911-914 (2005). 
[5] Campbell, C. L., Wood, K., Valentine, R. M., Brown, C. T. A., and Moseley, H., "Monte carlo modelling of daylight activated photodynamic therapy.," Physics in Medicine and Biology (in press).

[6] Wiegell, S., Hadersdal, M., Philipsen, P., Eriksen, P., Enk, C., and Wulf, H., "Continuous activation of ppix by daylight is as effective as and less painful than conventional photodynamic therapy for actinic keratoses; a randomized, controlled, single-blinded study," British Journal of Dermatology 158(4), 740-746 (2008).

[7] Morton, C., Wulf, H., Szeimies, R., Gilaberte, Y., Basset-Seguin, N., Sotiriou, E., Piaserico, S., Hunger, R., Baharlou, S., Sidoroff, A., and Braathen, L. R., "Practical approach to the use of daylight photodynamic therapy with topical methyl aminolevulinate for actinic keratosis: a european consensus," Journal of the European Academy of Dermatology and Venereology, n/a-n/a (2015).

[8] Zhu, C. and Liu, Q., "Review of monte carlo modeling of light transport in tissues," Journal of Biomedical Optics 18(5), 050902-050902 (2013).

[9] Prahl, S., Keijzer, M., Jacques, S., and Welch, A., "A monte carlo model of light propagation in tissue," in [SPIE Proceedings of Dosimetry of Laser Radiation in Medicine and Biology], 102-111, Press (1989).

[10] Takanori Igarashi, K. N. and Nayar, S. K., "The appearance of human skin: A survey," Foundations and Trends in Computer Graphics and Vision 3(1), 1-95 (2007).

[11] Farage, M., Miller, K., and Maibach, H., "Degenerative changes in aging skin," in [Textbook of Aging Skin], Farage, M., Miller, K., and Maibach, H., eds., 25-35, Springer Berlin Heidelberg (2010).

[12] Valentine, R., Brown, C. T. A., Moseley, H., Ibbotson, S., and Wood, K., "Monte carlo modeling of in vivo protoporphyrin ix fluorescence and singlet oxygen production during photodynamic therapy for patients presenting with superficial basal cell carcinomas," Journal of Biomedical Optics 16(4), 048002-048002-11 (2011).

[13] Valentine, R. M., Ibbotson, S., Wood, K., Brown, C., and Moseley, H., "Modelling fluorescence in clinical photodynamic therapy," Photochem. Photobiol. Sci. 12, 203-213 (2013).

[14] Raschke, C. and Elsner, P., "Skin aging: A brief summary of characteristic changes," in [Textbook of Aging Skin], Farage, M., Miller, K., and Maibach, H., eds., 37-43, Springer Berlin Heidelberg (2010).

[15] Iglesias-Guitian, J. A., Aliaga, C., Jarabo, A., and Gutierrez, D., "A biophysically-based model of the optical properties of skin aging," Computer Graphics Forum (EUROGRAPHICS 2015) To Appear (2015).

[16] Waller, J. M. and Maibach, H. I., "Age and skin structure and function, a quantitative approach (i): blood flow, ph, thickness, and ultrasound echogenicity," Skin Research and Technology 11(4), 221-235 (2005).

[17] Gilchrest, B. and Szabo, G., "Effects of ageing and chronic sun exposure on melanocytes in human skin," Journal of Investigative Dermatology 73(2), 141-143 (1979).

[18] J, C., K, Y., M, L., and et al, "Differential effects of photoaging vs intrinsic aging on the vascularization of human skin," Archives of Dermatology 138(11), 1437-1442 (2002).

[19] Karsten, A. E. and Smit, J. E., "Modeling and verification of melanin concentration on human skin type," Photochemistry and Photobiology 88(2), 469-474 (2012).

[20] Yoon, G., Welch, A., Motamedi, M., and Gemert, M., "Development and application of three-dimensional light distribution model for laser irradiated tissue," Quantum Electronics, IEEE Journal of 23, 1721-1733 (Oct 1987).

[21] Jacques, S. and Wang, L., "Monte carlo modeling of light transport in tissues," in [Optical-Thermal Response of Laser-Irradiated Tissue], Welch, A. and Van Gemert, M., eds., Lasers, Photonics, and Electro-Optics, 73-100, Springer US (1995).

[22] Wood, K. and R. J. Reynolds, R., "A model for the scattered light contribution and polarization of the diffuse h galactic background," The Astrophysical Journal 525(2), 799 (1999).

[23] Flock, S. T., Wilson, B. C., and Patterson, M. S., "Total attenuation coefficients and scattering phase functions of tissues and phantom materials at 633 nm," Medical Physics 14(5), 835-841 (1987).

[24] Valentine, R., "Biological aspects of photodynamic therapy," Ph.D. Thesis, University of St Andrews (2011).

[25] Michels, R., Foschum, F., and Kienle, A., "Optical properties of fat emulsions," Opt. Express 16, 5907-5925 (Apr 2008).

[26] Jacques, S., Joseph, R., and Gofstein, G., "How photobleaching affects dosimetry and fluorescence monitoring of pdt in turbid media," Proc. SPIE 1881, 168-179 (1993). 
[27] Farrell, T., Hawkes, R., Patterson, M., and Wilson, B., "Modeling of photosensitizer fluorescence emission and photobleaching for photodynamic therapy dosimetry," Appl. Opt. 37, 7168-7183 (Nov 1998).

[28] Jacques, S., "Optical properties of biological tissues: a review," Physics in Medicine and Biology 58(11), R37 (2013).

[29] Yudovsky, D. and Pilon, L., "Rapid and accurate estimation of blood saturation, melanin content, and epidermis thickness from spectral diffuse reflectance," Appl. Opt. 49, 1707-1719 (Apr 2010).

[30] Yudovsky, D. and Pilon, L., "Retrieving skin properties from in vivo spectral reflectance measurements," Journal of Biophotonics 4(5), 305-314 (2011).

[31] Henyey, L. and Greenstein, J., "Diffuse radiation in the Galaxy," 93, 70-83 (Jan. 1941).

[32] Van Gemert, M., Jacques, S., Sterenborg, H., and Star, W. M., "Skin optics," Biomedical Engineering, IEEE Transactions on 36(12), 1146-1154 (1989).

[33] Usami, A. and Kawasaki, N., "Modeling of solar spectral irradiance data from cloudless to overcast skies," Japanese Journal of Applied Physics 51, 10NF06 (2012).

[34] Bird, R. and Riordan, C., "Simple Solar Spectral Model for Direct and Diffuse Irradiance on Horizontal and Tilted Planes at the Earth's Surface for Cloudless Atmospheres," Journal of Applied Meteorology 25, 87-97 (1986).

[35] Lister, T., Wright, P., and Chappell, P., "Optical properties of human skin," Journal of Biomedical Optics 17(9), 090901-1-090901-15 (2012). 